\title{
Biomimetic soft matter
}

Article

Accepted Version

Hamley, I. (2011) Biomimetic soft matter. Soft Matter, 7 (20). pp. 9533-9534. ISSN 1744-683X doi:

https://doi.org/10.1039/c1sm90064a Available at https://centaur.reading.ac.uk/23772/

It is advisable to refer to the publisher's version if you intend to cite from the work. See Guidance on citing.

Published version at: http://dx.doi.org/10.1039/c1sm90064a

To link to this article DOI: http://dx.doi.org/10.1039/c1sm90064a

Publisher: Royal Society of Chemistry

All outputs in CentAUR are protected by Intellectual Property Rights law, including copyright law. Copyright and IPR is retained by the creators or other copyright holders. Terms and conditions for use of this material are defined in the End User Agreement.

\section{www.reading.ac.uk/centaur}

\section{CentAUR}

Central Archive at the University of Reading

Reading's research outputs online 


\section{Biomimetic Soft Matter}

\section{Guest Editorial - Ian Hamley}

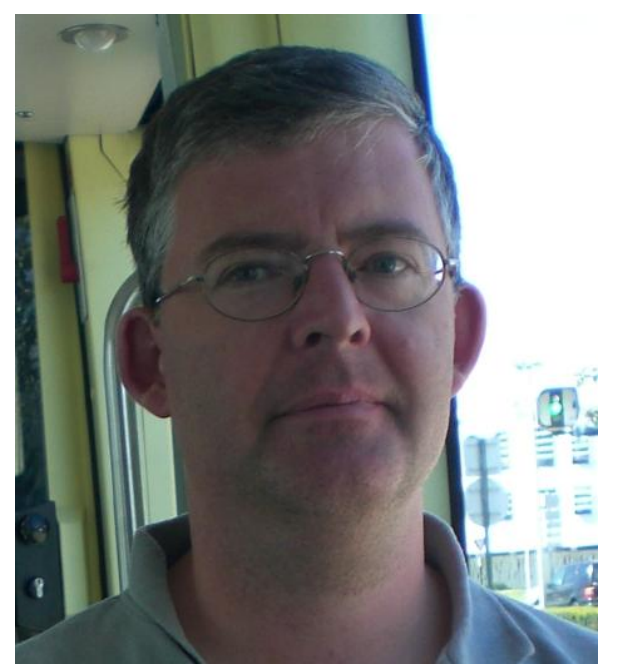

Biomaterials are often soft materials. There is now growing interest in designing, synthesizing and characterising soft materials that mimic the properties of biological materials such as tissue, proteins, DNA or cells. Research on biomimetic soft matter is therefore a developing theme with important emerging applications in biomedicine including tissue engineering, diagnostics, gene therapy, drug delivery and many others. There are also important basic science questions concerning the use of concepts from colloid and polymer science to understand the self-assembly of biomimetic soft materials. This issue of Soft Matter presents a selection of extremely topical articles on a diversity of biomimetic soft matter systems. I thank the contributors for this quite remarkable collection of papers, which report many fascinating discoveries and insights.

Stupp and coworkers describe an enzyme-responsive peptide amphiphile (PA) system that undergoes reversible self-assembly in response to reversible phosphorylation and dephosphorylation, phosphorylation being achieved via incorporation of a substrate for protein kinase A. Cleavage of phosphate groups using a phosphatase leads to the formation of filamentous structures and the concomitant triggered release of encapsulated anti-cancer drug doxorubicin. The theme of PA self-assembly is also explored by Löwik and coworkers. They report on the thermal stability and alignment under flow of a PA containing an octapeptide sequence derived from the CS protein of the malaria parasite Plasmodium falciparum. The temperature dependence of the critical aggregation concentration enables the thermodynamic parameters of fibrillisation to be quantified. Flow-induced birefringence reveals fracture at moderate shear rates. Tirrell and coworkers review peptide amphiphile self-assembly, with a focus on systems that remain soluble under physiological conditions. Whilst most PAs self-assemble into fibrillar systems, this group has designed PA assemblies that form spherical micelles.

Hudalla and Murphy review the development of self-assembled monolayers (SAMs) as mimics of the extracellular matrix (ECM). They discuss several methods to prepare 
SAM-based culture substrates via covalent and noncovalent chemistries and they review the SAM-based presentation of ECM-derived biomolecules to cells. Naumann et al. describe the development of planar lipid membranes including tethered bilayers based on peptide- or oligo-oxyethylene (OEO) tethers and protein-tethered bilayer lipid membrane (ptBLM). In the latter case, the activity of an immobilized enzyme was assayed and different conformational states depending on activation were observed.

Qi and coworkers make a nice contribution to the field of biomineralization in their report on templated silica structures which resemble microscale ceramic pots. A mechanism based on the helical coiling of silica fibres is proposed. Biomineralization is also the theme of the paper by Cui and coworkers. They have designed a peptide able to remineralize human dentine. The peptide incorporates calcium phosphate and collagen binding sequences. Both are required in successful remineralisation of calcium templated by the collagen matrix. The peptides direct the nucleation and growth of apatite crystals. Sommerdijk and coworkers have performed the biomimetic synthesis of calcium carbonate and investigated nucleation, growth and polymorph selection using acidic amino acid based copolymers with varying Glu/Asp/Ala ratios.

The development of synthetic mucosa-mimetic hydrogels to replace animal experimentation in characterisation of mucoadhesive drug delivery systems is reported by Khutoryanskiy and coworkers. The adhesion on synthetic hydrogels was measured and compared to that on buccal mucosa.

Agut et al. describe a new type of responsive peptide-containing diblock copolymer. A transition from vesicles to core-shell particles is induced upon addition of hydrophobic iron oxide nanoparticles into the hydrophobic domain in the vesicle wall. These particles undergo depletion-induced microphase separation and this drives a transition such that they form aggregate particles with a copolymer shell. Caruso and coworkers describe the PEGylation of so-called capsosomes, which are polymer hydrogel particles containing intact liposomal subcompartments, i.e. a shell comprising liposomes. These compartmentalized particles are useful in the development of cell-mimics. The aim of the PEGylation is to improve the stability of the liposomes against enzymatic degradation as characterized through detailed measurements in the presence and absence of BSA.

The helix-coil transition in a polymer-peptide conjugate can be triggered by addition of calcium ions, as discussed in the contribution from Börner and coworkers. They designed a conjugate containing PEG and a heptapeptide repeat peptide sequence incorporating multiple glutamic acid residues to confer concentration-dependent calcium binding properties. The self-assembly of PEG-peptide conjugates containing a designed helix-forming domain containing a histidine tag and a helical alanine-rich, glutamic-acid-containing domain, which exhibits $\mathrm{pH}$-responsive assembly behaviour has been investigated by Top et al. The effect of PEG molar mass was also examined.

Vollrath and coworkers discuss attempts to prepare mimics of spider silks, which have unique mechanical properties as elegantly reviewed in their contribution. This provides a framework to rationally develop methods to process artificial silk materials with the desired tensile properties. Zhang et al. describe a method to create silk fibroin nanoparticles functionalized with L-asparaginase. The enzyme immobilized in 
the silk fibroin nanoparticles showed enhanced stability and its activity was measured. These bioconjugates may have potential in the development of drug delivery systems.

The synthesis and self-assembly of polycarbonate-based hydrogels containing guanidine units and carboxylic acid units is described by Bartolini et al. Ring-opening polymerization methods are used to create the copolymers. As well as PEG crosslinking, electrostatic interactions between the functional moieties leads to novel $\mathrm{pH}$ responsive bio-inspired materials.

Artzner and coworkers review the self-assembly of peptide nanotubes, with a particular focus on the octapeptide lanreotide, as studied by that group. This system and its anaologues provide a wealth of data on the self-assembly mechanisms, and kinetic and thermodynamic aspects. The use of these peptides to template inorganic nanotubes is also described.

Adams and coworkers describe how the mechanical properties of Fmoc-dipeptide gels may be controlled through use of cosolvent and temperature processing conditions. These fibril-forming low molecular weight gelators are investigated in the presence of DMSO under conditions similar to those used in cell culture and controlled release applications.

Payne and coworkers review biologically active phenolics, and their function. These molecules have a remarkable range of roles in a variety of materials. Their primary focus is on incorporation of phenolics into chitosan films to provide new properties.

Takeuchi and coworkers have developed a fluorescence-based system for protein assays based on quantum dots functionalized by an imprinting method based on liquid phase deposition of $\mathrm{TiO}_{2}$ on surface- immobilized ribonuclease A. Subsequent removal of protein leaves protein binding sites available for the fluorescence quenching measurements.

Fratzl and coworkers provide a highly topical review on the interactions between cells and substrates in the context of tissue development. The constraints provided by such interactions, and geometrical constraints lead to emergent properties not possessed by individual cells, such as the long-range organization of the ECM.

A new method to create giant unilamellar vesicles using localised infrared laser heating is introduced by Billerit et al. These systems are widely used as cell membrane mimics, and this technique complements existing methods of vesicle production with its own unique advantages.

An interesting new type of amphiphilic biomaterial is described by Mendes et al. They have prepared polysaccharide amphiphiles, via palmitoylation of xanthan. Under appropriate conditions, self-assembled microcapsules are observed and these are used to encapsulate cells. 